\title{
Interaction of non-adherent suspended neutrophils to complement opsonized pathogens: a new assay using optical traps
}

\author{
Tomoko Suzuki ${ }^{1}$, Masaru Yanai ${ }^{1}$, Hiroshi Kubo ${ }^{1}$, Akio Kanda ${ }^{1}$, Hidetada Sasaki ${ }^{1}$, James P Butler ${ }^{1,2}$ \\ ${ }^{1}$ Department of Geriatric and Respiratory Medicine, Tohoku University School of Medicine, Sendai 980-8574, Japan; ${ }^{2}$ Physiology \\ Program, Harvard School of Public Health, Boston, MA 02115, USA
}

Phagocytosis of opsonized pathogens by circulating non-adherent neutrophils is an essential step in host defense, which when overwhelmed contributes to sepsis. To investigate the role played by ligation of complement receptors CR3 and CR4 in non-adherent neutrophils, we designed a novel assay system utilizing dual optical traps, respectively, holding a suspended unactivated cell and presenting a specific ligand-coated bead to the cell surface. We chose anti-CD18 as an example ligand, mimicking the bacterial opsonizing complement fragment iC3b. Presentation of anti-CD18-coated beads elicited both pseudopodial protrusion and subsequent phagocytosis. This is in sharp contrast to previously reported responses of adherent neutrophils, which phagocytize opsonized particles without pseudopod formation. We used this same new assay to probe actomyosin pathways in the neutrophil's pseudopodial and phagocytic response. Disruption of actin or inhibition of myosin light-chain kinase dose-dependently reduced pseudopod formation and phagocytosis rates. In summary, i) the new dual trap assay can be used to study the responses of suspended neutrophils to a variety of ligands, and ii) in a first application of this technique, we found that local ligation of CR3/4 in unactivated neutrophils in suspension induces pseudopod formation and phagocytosis at that site, and that these events occur via an actomyosindependent pathway.

Cell Research (2006) 16:887-894. doi:10.1038/sj.cr.7310103; published online 24 October 2006

Keywords: neutrophil, phagocytosis, pseudopod formation, actomyosin, complement, CR3/4 receptor

\section{Introduction}

Sepsis remains a serious cause of morbidity and mortality worldwide, and is associated with a systemic inflammatory response to circulating bacteria in the bloodstream, one of the critical features of the septic condition [1]. A key feature of host defense is phagocytosis of these pathogens by neutrophils, for which opsonization of bacteria by complement fragments, $\mathrm{C} 3 \mathrm{~b}$ and $\mathrm{iC} 3 \mathrm{~b}$, is an important first step. Leukocyte $\beta_{2}$-integrins, CD11b/CD18 and $\mathrm{CD} 11 \mathrm{c} / \mathrm{CD} 18$, are $\mathrm{iC} 3 \mathrm{~b}$ receptors $(\mathrm{CR} 3: \mathrm{CD} 11 \mathrm{~b} / \mathrm{CD} 18$; CR4: CD11c/CD18), and are constitutively and inductively expressed on the cell surface of leukocytes. $\beta_{2}$-integrins are involved in virtually every aspect of leukocyte func-

Correspondence: Hiroshi Kubo

Tel: +81-22-717-7182; Fax: +81-22-717-7186;

E-mail: hkubo@geriat.med.tohoku.ac.jp

Received 27 June 2006; revised 17 September 2006; accepted 21 September 2006; published online 24 October 2006 tion, including adhesion and transmigration through the endothelial cells, the immune response, phagocytosis of pathogens, and leukocyte activation $[2,3]$. However, most of what is known about $\beta_{2}$-integrin-induced leukocyte function, especially at the individual cell level, has been based on experiments using adherent and activated cells, which may or may not conform to the behavior of the circulating cells important in sepsis.

In order to address this issue, and to investigate the response of circulating, unactivated neutrophils on an individual cell basis, we developed a novel assay system, wherein ligand-coated beads can be presented to individual neutrophils suspended in medium. The assay is based on a dual laser tweezer trap system: one trap holds the suspended neutrophil, and the other holds a ligand-coated bead under the control of a joystick used for presentation of the ligand to the cell surface. This system can be generalized to a wide class of ligands that can be coated onto beads. For our purposes, we chose the anti-CD18 molecule as 
a representative ligand, as it mimics part of complement fragment $\mathrm{iC} 3 \mathrm{~b}$, one of the important bacterial opsonizing molecules in the promotion of phagocytosis.

In addition to the issues of ligand binding, the molecular basis for mechanical cellular response in the neutrophil is not yet completely understood, particularly with respect to pseudopodial protrusion and phagocytosis. It is well established that myosin-dependent pathways are important for phagocytosis and for retraction [4] of the trailing region of adherent locomoting cells, but the extent to which actomyosin interactions, in particular, are important to pseudopod formation or phagocytosis in non-adherent cells remains an open question. Using a variety of pharmacologic interventions, we therefore assessed the importance of actomyosin-dependent pathways in both pseudopod protrusion and subsequent phagocytic responses of neutrophils to the presentation of anti-CD18-coated beads.

\section{Materials and Methods}

\section{Cell preparation}

Neutrophils were isolated from venous blood of 10 normal human volunteers (eight males and two females, $33.8 \pm 6.1$ years old) drawn from the faculty of the Department of Geriatric and Respiratory Medicine, by a density gradient technique [5], and suspended in RPMI 1640 medium. Verbal informed consent was obtained from each subject. A round and floating neutrophil was selected and held by one optical trap (see below). An antibody-coated polystyrene microbead, caught in a second optical trap, was brought up and attached to the cell.

\section{Bead preparation}

Carboxylated polystyrene microbeads ( $3 \mu \mathrm{m}$ diameter) were obtained from Polyscience Inc. (Warrington, PA, USA). These microbeads bearing rat anti-mouse IgG1 monoclonal antibody ( $\mathrm{mAb}$ ) (Pharmingen, San Diego, CA, USA), covalently coupled to the bead by the carbodiimide method [6] (control beads), were used for control experiments. Identically prepared beads were then incubated with anti-human CD18 mAb (isotype: mouse IgG1; Endogen, Woburn, MA, USA), and these (denoted anti-CD18 beads) were used for test experiments. Both control and anti-CD18 beads were rinsed three times with phosphate-buffered saline (without calcium) prior to the experiments.

\section{DIC microscopy with optical tweezers}

A schematic of the experimental apparatus is shown in Figure 1. Samples were observed under a differential interference contrast microscope (Leica DM-IRB; Leica Microsystems, Wetzlar, Germany) with a $100 \times$ oil-immersion objective lens, equipped with a videoenhancing system. Dual linearly polarized laser beams from an $\mathrm{Nd}$ : YAG laser emitting at $1064 \mathrm{~nm}$ were introduced into the epifluorescence port of the microscope to make two optical traps (Sigma Koki,

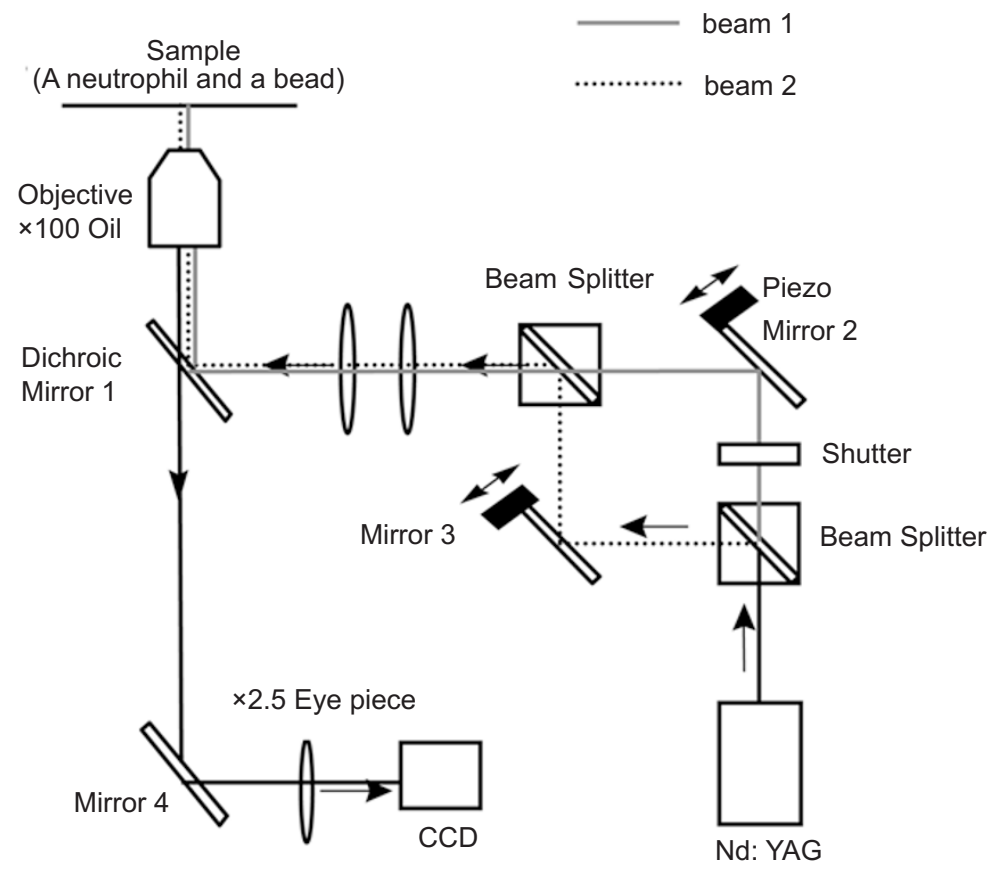

Figure 1 A schema for the micromanipulation system. Beam 1 (gray line) is freely moveable by the piezo drive-controlled Mirror 2 , connected with the x-y joystick, and is used to grab a bead. Beam 2 (dotted line) is used to trap a neutrophil. Samples are observed with the CCD video camera. 
Saitama, Japan). The position of one trap (sample termination of the dotted line in Figure 1), which was used to hold a floating non-adherent neutrophil, was fixed. The other trap was moveable (sample termination of the gray line in Figure 1); it was used to trap a bead, which could then be manipulated by an $x-y$ joystick connected to the piezo drive-controlled Mirror 2 (Figure 1).

\section{Inhibitory experiments}

To determine the effect of inhibition of myosin light-chain kinase (MLCK), myosin ATPase activity, and actin or microtubule polymerization on pseudopod formation and phagocytosis mediated by presentation of an anti-CD18-coated bead, a series of experiments with inhibitory compounds were performed. For the actomyosindependent pathway experiments, neutrophils in suspension were pretreated on ice for $30 \mathrm{~min}$ with the MLCK enzymatic inhibitors ML-9 (1-(5-chloronaphthalene-1-sulfonyl)-1H-hexahydro-1,4-diazepine hydrochloride) or ML-7 (1-(5-Iodonaphthalene-1-sulfonyl)$1 \mathrm{H}$-hexahydro-1,4-diazepine hydrochloride) $(1,3,10$, and $30 \mu \mathrm{M}$; Seikagaku, Tokyo, Japan), or with the myosin ATPase inhibitor butanedione monoxime (BDM) (1, 3, 7, 10, and 20 mM; Sigma, St Louis, MO, USA). Actin microfilaments and microtubules were, respectively, disrupted with cytochalasin D (10 $\mu \mathrm{M}$; Sigma) or nocodazole (30 $\mu \mathrm{M}$; Sigma). Then, either anti-CD18 beads or control beads suspended in the medium containing the identical concentration of each drug was added to the pretreated cells.

\section{Ligation and control experiments on mechanical stimula- tion}

To determine if receptor clustering is necessary, presumably through at least dimerization, we studied cells in medium containing anti-CD18 antibody in solution $(20 \mu \mathrm{g} / \mathrm{ml})$, which is sufficient to saturate the receptors without inducing ligation. An anti-CD18 antibody-coated bead was subsequently presented to the cell, and the pseudopodial protrusion or phagocytic response was measured. As a control against the possibility that simple mechanical stimulation associated with bead presentation might induce pseudopodial protrusion or bead phagocytosis, we repeated these experiments with anti-IgG1-coated beads in control medium.

\section{Protocol}

Cells were observed on a video display screen, and videotape recordings were made for later analysis. During playback, the time of initial contact of the presenting bead with the cell was noted, and the behavior of the cell was followed for at least 5 min following bead presentation. If the cell protruded a pseudopod and had directional cytoplasmic flow into the pseudopod, or internalized the bead, we recorded the time lapse of these cellular events. The initial pseudopodial protrusion at the bead site was clearly visible on the display screen; the recorded time lapse was accurate to within a few seconds. Similarly, the engulfment of the bead if a phagocytic event took place was equally abrupt and visible. Experimental runs were terminated following pseudopod formation or phagocytosis of the bead at any time within the 5-min observation period, or if no response was observed over the 5-min period.

Results were expressed as an effective rate constant for the initiation of either a pseudopodial protrusion event or for phagocytosis, given by the reciprocal of the respective time intervals. This facilitates the description of data where no events were noted in the experimental time period of $5 \mathrm{~min}$. These were arbitrarily assigned a rate constant value of $0.2 \mathrm{~min}^{-1}$ (the reciprocal of our maximum observation period), recognizing that this is therefore an upper bound for the rate when no event was observed. Specifically, let be the time interval from the bead presentation to either a pseudopodial protrusion or a phagocytic event, or $5 \mathrm{~min}$ if no event took place. We define the rate for that event by

$$
r=\left\{\begin{array}{l}
1 / \Delta t, \Delta t<5 \mathrm{~min} \\
0.2 \mathrm{~min}^{-1}, \Delta t=5 \mathrm{~min}
\end{array}\right.
$$

these are the experimental values used in all data analyses. To justify this approach, we note that if the probabilistic structure of the stochastic process determining each realization of a pseudopodial protrusion or phagocytic event were known, then there would be no ambiguity in defining an appropriate model and statistical test for analysis of the observed independent time intervals between bead presentation and cellular response. However, to our knowledge, this response probability function is unknown, both within a given cell and across different cells. We have therefore adopted a conservative descriptive language for our observations, and expressed all time intervals by their respective inverses as rate constants. Finally, as noted above, uncertainty in the time intervals between bead presentation and pseudopod formation or phagocytosis was a few seconds at most; this has a negligible effect on the rates reported here.

\section{Statistics}

All data are reported as means $\pm \mathrm{SD}$, and significance is taken at $p<0.05$ or $<0.001$ (see Figure legends). No significant differences were found between cells from different donors. Results for all experiments were therefore pooled by protocol and intervention, each $N$ of which thus represents multiple cells from multiple donors. Dose/response comparisons were made with Student's $t$-test; relationships between PP rates and Phago rates for the various experimental conditions were examined with standard linear regression, for which the correlation coefficients $r^{2}$ and significance levels were computed. Note that we have included the rate constant of $0.2 \mathrm{~min}^{-1}$ for those experiments in which no event took place during the 5-min observation period. It is legitimate to question whether these points should be included in the regression analyses or not. Indeed, some difficulties in data interpretation associated with the assignment of an upper limit on $r$ when no event was observed could be avoided by simply excluding any cells that did not respond, but this results in a biased statistical analysis due to data censorship, the effects of which are unknown in these circumstances. Moreover, an examination of the data shown in Figures 3-5 shows that replacing this upper bound on the rate constant by any other number (such as zero for no event) would have a negligible effect on our conclusions. In any event, the adoption of an upper bound for the rate constants associated with null observations is the conservative approach which we prefer.

\section{Results}

Anti-CD18 bead presentation induced pseudopod formation and subsequent cytoplasmic flow at the presentation site in 64 out of 64 cells in the experiments in the absence of cytoskeletal disruptors, with respective rates of $0.89 \pm 0.38$ and $0.66 \pm 0.26 \mathrm{~min}^{-1}($ mean $\pm \mathrm{SD})$. These correspond to time intervals of $78 \pm 27 \mathrm{~s}$ and $101 \pm 30 \mathrm{~s}$ following anti-CD18 
bead presentation. A representative photomicrograph set is shown in Figure 2. The sequence of events is as follows: bead presentation at time 0 , protrusion of a small process at $80 \mathrm{~s}$, beginning of cytoplasmic streaming at $95 \mathrm{~s}$, and phagocytosis of the bead in the advancing pseudopod at 105 s. In Supplement 1, we present a video of the entire sequence of bringing an antibody-coated bead to the cell surface, showing its binding, the waiting period of quiescence, the formation of a pseudopod, and the subsequent phagocytosis of the bead (Supplement 1).

Figures 3-5 show the results of inhibiting the actomyosin-dependent pathways, with MLCK inhibitors ML-9 and ML-7, or with the myosin ATPase inhibitor BDM at the
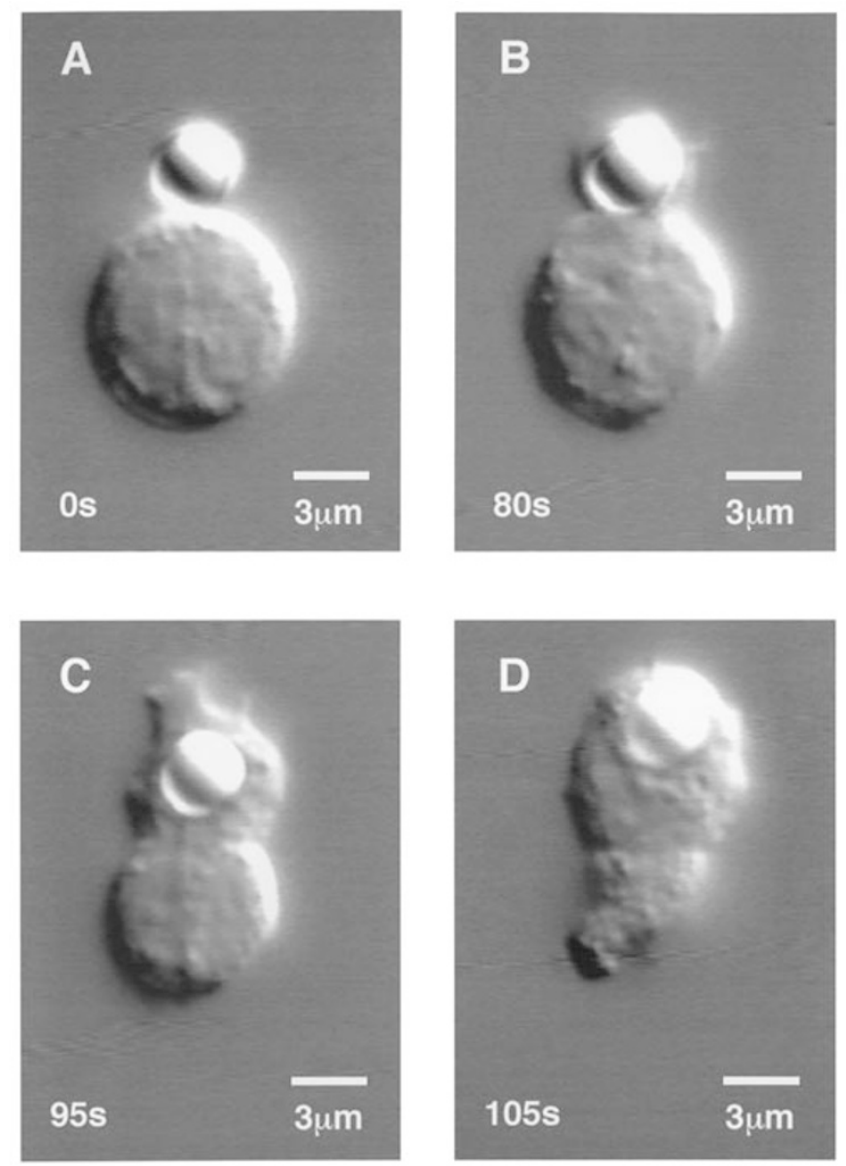

Figure 2 Photomicrographs of pseudopod formation and phagocytosis of an anti-CD18 bead at the presentation site. A: An anti-CD18 bead was attached to a round non-adherent neutrophil suspended in the control medium by an optical trap. B: $80 \mathrm{~s}$ later, the cell protruded a small process at the presentation site (this time interval was used for computing the PP rate, in this case $\left.1 / 80 \mathrm{~s}=0.75 \mathrm{~min}^{-1}\right)$. C: The cell continued to protrude a pseudopod followed by cytoplasmic streaming (95 s). D: At $105 \mathrm{~s}$, the cell finally phagocytized the bead in the advancing pseudopod (this time was used for computing the Phago rate, in this case $1 / 105 \mathrm{~s}=0.57 \mathrm{~min}^{-1}$ ).
A

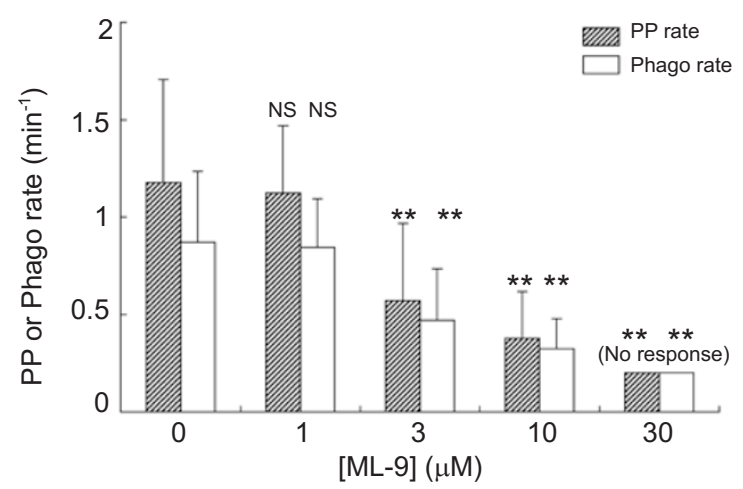

B

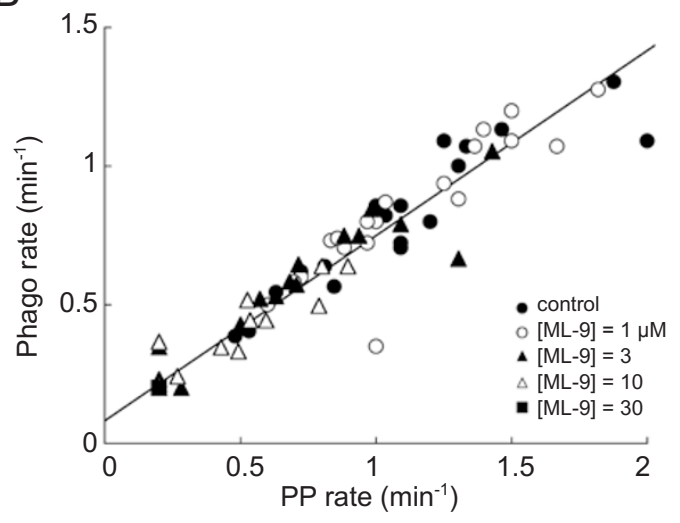

Figure 3 (A) ML-9 dose/response relationship for pseudopod formation rate (PP rate) and phagocytosis rate (Phago rate) of an anti-CD18 bead presented to the cell surface. A rate of $0.2 \mathrm{~min}^{-1}$ denotes no event recorded in the $5 \mathrm{~min}$ observation period (an upper bound for the true rate for that cell). * and $* *$ denote $p<0.05$ and 0.001 , respectively, in comparisons to untreated PP and Phago rates. Numbers of cells studied with increasing dose, beginning with no treatment, are 19, 19, 20, 20, 18. (B) Phago rate vs. PP rate with varying doses of ML9. $N=94$, regression equation: Phago rate $=0.67 \mathrm{PP}$ rate $+0.08 \mathrm{~min}^{-1}$, $r^{2}=0.94, p<0.001$.

indicated concentrations. In each figure, panel A shows the dose-dependent inhibition of pseudopod formation and phagocytosis of an anti-CD18 antibody-coated bead. Panel B in each figure shows the relationship between the pseudopod formation rate and phagocytosis rate. In all cases, rates of $0.2 \mathrm{~min}^{-1}$ indicate that no event had occurred within the 5-min observation period. The $\mathrm{ED}_{50}$ for ML-9 inhibition of anti-CD18 bead-induced pseudopod formation and phagocytosis was about $3 \mu \mathrm{M}$; the corresponding $\mathrm{ED}_{50}$ for ML-7 was about $10 \mu \mathrm{M}$. The $\mathrm{ED}_{50}$ for BDM was about $3 \mathrm{mM}$.

Actin and microtubules were, respectively, disrupted by pretreatment with cytochalasin $\mathrm{D}$ or nocodazole. Ten 
A

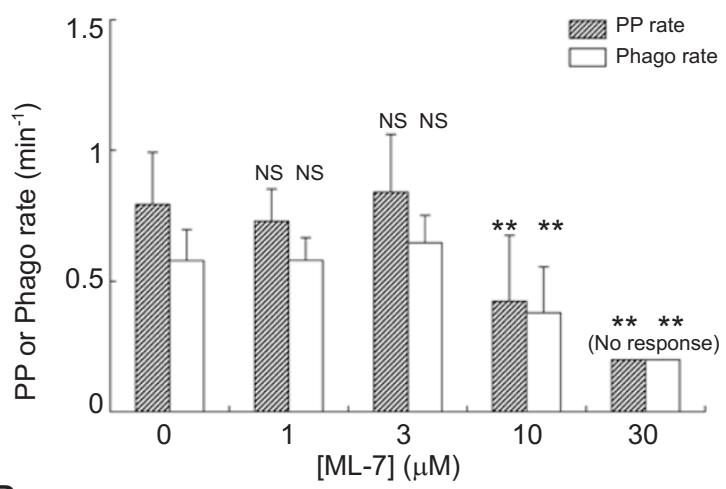

B

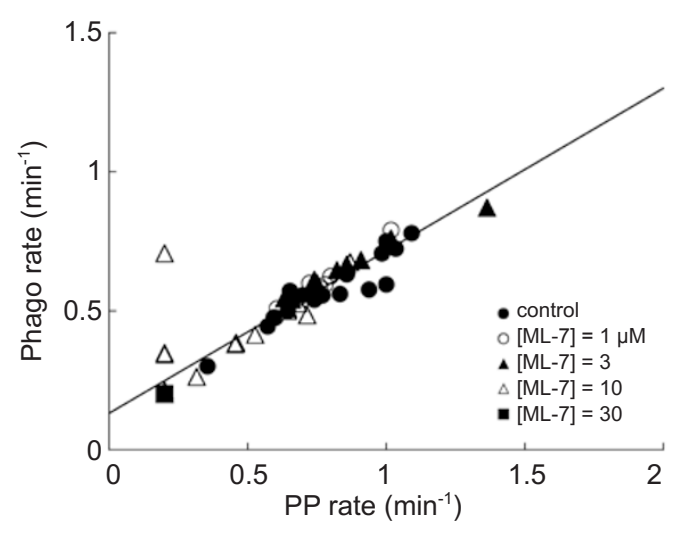

Figure 4 (A) As in Figure 3, dose/response relationship with ML7 treatment. $N=17,10,10,20,6$ for the increasing doses. (B) As in Figure 3, PP vs Phago rate relationships with ML-7 treatment. $N=61$, regression equation: Phago rate $=0.58 \mathrm{PP}$ rate $+0.13 \mathrm{~min}^{-1}$, $r^{2}=0.85, p<0.001$.

micromolar cytochalasin D completely blocked both pseudopod formation and phagocytosis of an anti-CD18 antibody-coated bead (data not shown), whereas $30 \mu \mathrm{M}$ nocodazole had no effect on pseudopod formation or phagocytosis rates.

Figure 6 shows the effect of the presence of anti-CD18 antibody in the medium. There was a dramatic fall in the response of the cells to presentation of anti-CD18 beads, confirming that ligation of CD18 molecules is necessary for the activation of pseudopodial and phagocytic responses. Control anti- $\mathrm{IgG}_{1}$-coated beads did not induce pseudopod formation or bead phagocytosis in any experiments in control medium. This remained true for cells pretreated with cytochalasin D or nocodazole and for cells following the administration of ML-9 or ML-7. This confirms that the cellular response we observed was not associated with mechanical stimulation caused by bead contact.
A

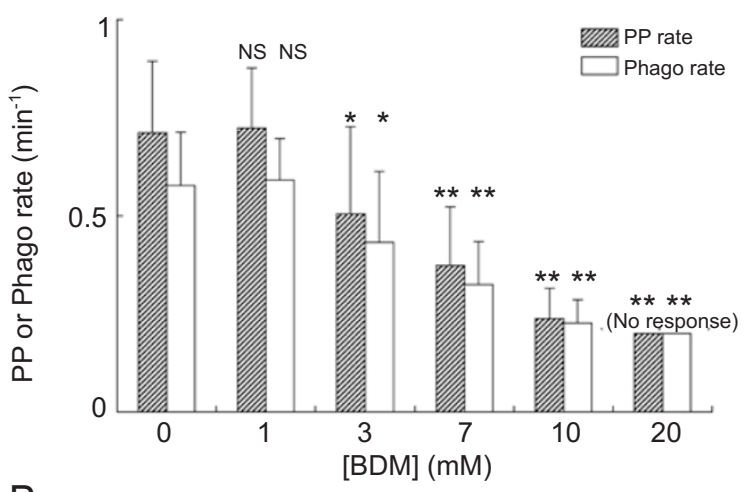

B

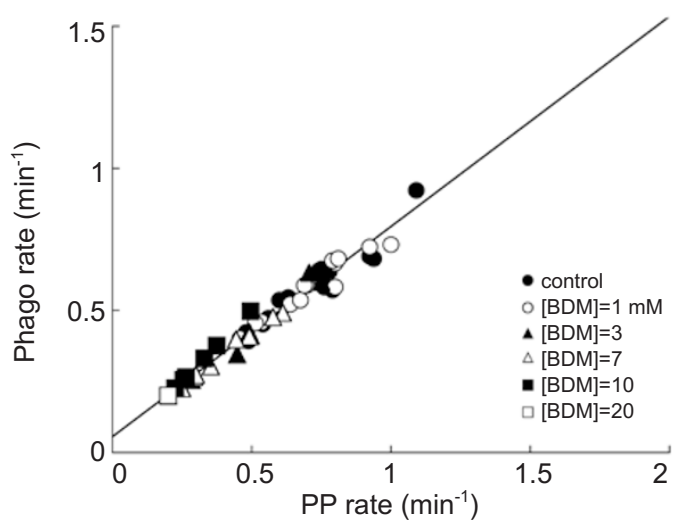

Figure 5 (A) As in Figure 3, dose/response relationship with BDM treatment. $N=14,14,10,10,20,10$ for the increasing doses. (B) As in Figure 3, PP vs Phago rate relationships with BDM treatment. $N=76$, regression equation: Phago rate $=0.74$ $\mathrm{PP}$ rate $+0.06 \mathrm{~min}^{-1}, r^{2}=0.98, p<0.001$.

\section{Discussion}

In this study, we investigated the regional response of non-adherent unactivated neutrophils to ligation of $\beta_{2}$-integrins, which is clearly of importance in understanding the responses of circulating neutrophils in clinical situations such as sepsis. In particular, circulating bacteria are opsonized by complement component $\mathrm{iC} 3 \mathrm{~b}$, and circulating neutrophils phagocytize them via the $\beta_{2}$-integrins, CR3 and CR4. However, virtually all previous studies have used adherent neutrophils to analyze $\beta_{2}$-integrins, the results of which are therefore not necessarily reflective of non-adherent neutrophils responding to sepsis. The observations reported here represent, to our knowledge, the first evidence suggesting that ligation of CD18 molecules in round, floating, non-adherent neutrophils directly induces pseudopod formation and bead phagocytosis, and that these events 


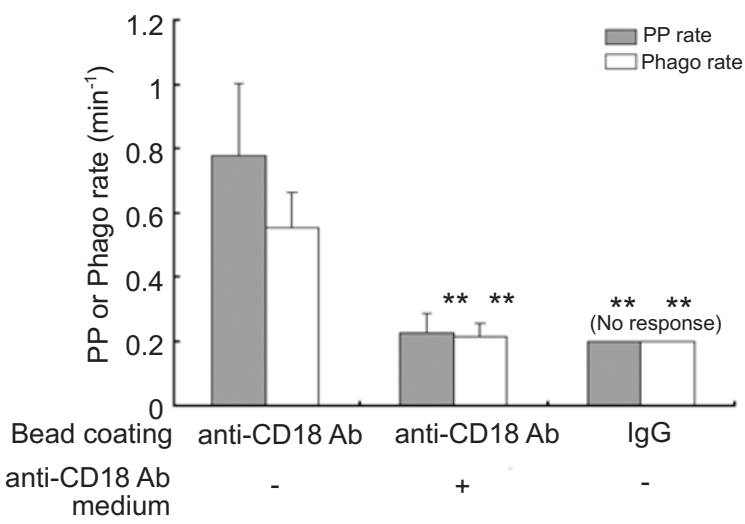

Figure $6 \mathrm{PP}$ and Phago rates for an anti-CD18 bead when anti-CD18 antibody is present in the medium $(N=27)$ compared with an antiCD18 bead in control medium $(N=14)$. Also shown is the observation $(N=20)$ that there was a complete lack of cellular response to mechanical effects associated with an anti- $\operatorname{IgG}_{1}$ coated bead presented to the cell in control medium.

are blocked by the inhibition of either MLCK or myosin ATPase activity. The latter finding strongly suggests that actomyosin pathways are necessary for pseudopodial protrusion locomotion and phagocytosis.

In addition, most previous methodologies have studied the combined effects of phagocytosis and chemotaxis, because they analyze phagocytosis as part of the process of chemotaxis with the cell moving towards the particle. Recently, Hallet et al. elegantly evaluated an isolated effect of the iC $3 b$-accelated phagocytosis of infecting pathogens by neutrophils using micromanipulation $[7,8]$. This strongly suggests a complementary role for our new assay in characterizing iC3b-accelated phagocytosis.

Two distinct mechanisms of phagocytosis have been described $[9,10]$. Type I phagocytosis occurs through pseudopodia extension and engulfment, and is mediated via FcyRs. Type II phagocytosis results from sinking of the opsonized particle intracellularly without membrane protrusion, and is mediated by complement receptor type 3 (CR3, Mac-1, $\alpha \mathrm{M} \beta 2$ ) in adherent $\mathrm{CHO}$ cells expressing human CR3 [10] and in macrophages [11]. CR3 is a heterodimeric transmembrane glycoprotein, belonging to the $\beta_{2}$-integrin family, consisting of CD11/CD18 [12], and it has been generally thought that type I phagocytosis (with pseudopodial protrusion) is not associated with $\beta_{2}$-integrins, at least in adherent cells. In contrast, and perhaps surprisingly, our results show that type I phagocytosis is universally seen in non-adherent suspended neutrophils presented with anti-CD18-coated beads. Although different cell types might well utilize different mechanisms of phagocytosis, it is nevertheless extremely interesting that such a difference should exist between the adherent activated cell and the circulating non-activated cell, pointing to the potential importance of $\beta_{2}$-integrins in sepsis.

With respect to mechanism, we previously hypothesized that development of intracellular pressure is the motive force of pseudopodial protrusion [13-15], which in this study was found to be a near universal precursor to phagocytosis. The results of the current study support this hypothesis, and appear to be inconsistent with the pseudopodial protrusion being the result of pushing (in compression) of polymerizing F-actin alone. Importantly, with the suspended non-adherent cells studied here, there can be, by definition, no cell-cell adhesions such as that through endothelial ICAM-1 or any interactions with extracellular matrix proteins, which might otherwise have contributed to pseudopodial protrusion. If pressure is indeed important for the precursor pseudopod formation, we asked whether this could be modulated through disruption of actomyosin pathways (note that swelling may also contribute to intracellular pressure [16]). Our results strongly suggest that this is the case. In particular, the $\mathrm{ED}_{50}$ 's for ML-9 and ML-7 that we observed for inhibition of pseudopod formation correspond roughly to similar values found by others for inhibition of MLCK activity [17, 18]. Similar correspondences were found with the myosin ATPase inhibitor BDM [17, 19, 20], although there is a question about its specificity with respect to non-muscle myosin II and its potential for interfering with acting polymerization [21]. Nevertheless, despite the potentially significant difference between activated and adherent vs quiescent and suspended cells, the close correspondence of our observed $\mathrm{ED}_{50} \mathrm{~s}$ supports the hypothesis that myosin-driven contraction is an essential pathway for pseudopod formation. Moreover, we note that adherent cells also show a time course for substrate phosphorylation in the 1-5 min range [18], which is strikingly similar to the rates we found for pseudopod formation and phagocytosis in non-adherent cells.

Finally, the observation that disruption of F-actin inhibited both pseudopod formation and phagocytosis comes as no surprise, as actin polymerization is known to be essential to these events [22, 23]. Somewhat surprisingly, however, we found that microtubule assembly plays little role in these events, consistent with the recent observations of Niggli et al. [24], and we suggest that whatever role played by microtubules in chemotactically induced locomotion may not be relevant to pseudopod formation in non-adherent cells.

We found considerable variation in pseudopod formation and phagocytosis rates among cells (note the standard deviations in Figures 3-5). Nevertheless, as shown in panel $\mathrm{B}$ of these figures, there was a striking correlation between 
Phago rates and PP rates, both within groups (controls or at any given level of drug administration) and for all data pooled. Cells that were dynamically fast responders in the sense of an early pseudopod formation were proportionately fast in phagocytosing the bead, and a similar trend is true for slow responders. This is evidence that the dynamics of pseudopod formation and phagocytosis are strongly linked, most likely through a common pathway. This is perhaps to be expected, but it is by no means necessary, as these are distinct cell functions (see, for example, the discussion above regarding the differences between type I and type II phagocytosis, wherein pseudopodial protrusion and phagocytosis are indeed seen as independent and distinct cell functions).

In summary, we have developed a novel assay for the study of non-adherent suspended leukocyte responses to a wide class of ligands, which may shed new light on host defenses in bacteremia and sepsis. This assay utilizes a dual laser trap system and ligand-coated beads; one trap is used to hold the suspended cell, and the other is used to present the coated bead to the cell surface. As a specific example of this new assay, we studied the response of suspended neutrophils to the presentation of anti-CD18-coated beads. Unlike adherent cells, we uniformly found a strong association between pseudopodial protrusion and phagocytosis (type I). Further studies utilizing pharmacologic interventions revealed that $\beta_{2}$-integrin-mediated pseudopod formation and phagocytosis are dependent on actomyosin contraction but not on microtubule assembly. This in turn is consistent with the hypothesis that contractile machinery in the body of the cell causes an increase in intracellular pressure, which in turn may be used as the motive force in the underlying mechanism driving pseudopodial protrusion and cytoplasmic flow, especially in the suspended cell where there is no extracellular matrix to bind in the promotion of locomotion and transendothelial migration. These findings may be of importance in developing new therapeutic approaches to patients with sepsis.

\section{Acknowledgments}

This work was supported by grants from the Japan Society for the Promotion of Science No. 17590776 to HK. We thank M Sato of Nambu-irika Co. for help in maintaining optimal operating conditions of the DIC microscope, lasers, and optical traps. This work was supported in part by Mombusho Grant No. 12670544, and a JAFAH fellowship from the Japan Foundation for Aging and Health (JPB).

\section{References}

1 Bone RC, Grodzin CJ, Balk RA. Sepsis: a new hypothesis for pathogenesis of the disease process. Chest 1997; 112:235-243.

2 Plow EF, Haas TA, Zhang L, Loftus J, Smith JW. Ligand binding to integrins. J Biol Chem 2000; 275:21785-21788.

3 Menegazzi R, Busetto S, Decleva E, Cramer R, Dri P, Patriarca P. Triggering of chloride ion efflux from human neutrophils as a novel function of leukocyte beta 2 integrins: relationship with spreading and activation of the respiratory burst. J Immunol 1999; 162:423-434.

4 Niggli V. Rho-kinase in human neutrophils: a role in signaling for myosin light chain phosphorylation and cell migration. FEBS Lett 1999; 445:69-72.

5 Kalmar JR, Arnold RR, Warbington ML, Gardner MK. Superior leukocyte separation with a discontinuous one-step FicollHypaque gradient for the isolation of human neutrophils. J Immunol Methods 1988; 110:275-281.

6 Okano K, Takahashi S, Yasuda K, Tokinaga D, Imai K, Koga M. Using microparticle labeling and counting for attomole-level detection in heterogeneous immunoassay. Anal Biochem 1992; 202:120-125.

7 Dewitt S, Hallett MB. Cytosolic free $\mathrm{Ca}^{2}+$ changes and calpain activation are required for beta-integrin-accelerated phagocytosis by human neutrophils. J Cell Biol 2002; 159:181-189.

8 Dewitt S, Laffafian I, Hallett MB. Phagosomal oxidative activity during beta-2 integrin (CR3)-mediated phagocytosis by neutrophils is triggered by a non-restricted $\mathrm{Ca}^{2}+$ signal: $\mathrm{Ca}^{2}+$ controls time not space. J Cell Sci 2003; 116:2857-2865.

9 Caron E, Hall A. Identification of two distinct mechanisms of phagocytosis controlled by different Rho GTPases. Science 1998; 282:1717-1721.

10 Le Cabec V, Carreno S, Moisand A, Bordier C, MoridonneauParini I. Complement receptor 3 (CD11b/CD18) mediates type I and type II phagocytosis during nonopsonic and opsonic phagocytosis, respectively. J Immunology 2002; 169:2003-2009.

11 Kaplan G. Differences in the mode of phagocytosis with Fc and C3 receptors in macrophages. Scand J Immunol 1977; 6:797807.

12 Sengelov H. Complement receptors in neutrophils. Crit Rev Immunol 1995; 15:107-131.

13 Yanai M, Kenyon CM, Butler JP, Macklem PT, Kelly SM. Intracellular pressure is a motive force for cell motion in Amoeba proteus. Cell Motil Cytoskeleton 1996; 33:22-29.

14 Yanai M, Butler JP, Suzuki T et al. Intracellular elasticity and viscosity in the body, leading, and trailing regions of locomoting neutrophils. Am J Physiol 1999; 277:C432-C440.

15 Yanai M, Butler JP, Suzuki T, Sasaki H, Higuchi H. Regional rheological differences in locomoting neutrophils. Am J Physiol Cell Physiol 2004; 287:C603-C611.

16 Zhelev DV, Hochmuth RM. Mechanically stimulated cytoskeleton rearrangement and cortical contraction in human neutrophils. Biophys J 1995; 68:2004-2014.

17 Eddy RJ, Pierini LM, Matsumura F, Maxfield FR. Ca²+-dependent myosin II activation is required for uropod retraction during neutrophil migration. J Cell Sci 2000; 113:1287-1298.

18 Mansfield PJ, Shayman JA, Boxer LA. Regulation of polymorphonuclear leukocyte phagocytosis by myosin light chain kinase after activation of mitogen-activated protein kinase. Blood 2000; 95:2407-2412.

19 Higuchi H, Takemori S. Butanedione monoxime suppresses contraction and ATPase activity of rabbit skeletal muscle. J Biochem 1989; 105:638-643. 
20 Urwyler N, Eggli P, Keller HU. Effects of the myosin inhibitor 2,3-butanedione monoxime (BDM) on cell shape, locomotion and fluid pinocytosis in human polymorphonuclear leucocytes. Cell Biol Int 2000; 24:863-870.

21 Ostap EM. 2,3-Butanedione monoxime (BDM) as a myosin inhibitor. J Muscle Res Cell Motil 2002; 23:305-308.

22 Condeelis J. Are all pseudopods created equal? Cell Motil Cy- toskeleton 1992; 22:1-6.

23 May RC, Machesky LM. Phagocytosis and the actin cytoskeleton. J Cell Sci 2001; 114:1061-1077.

24 Niggli V. Microtubule-disruption-induced and chemotactic-peptide-induced migration of human neutrophils: implications for differential sets of signaling pathways. J Cell Sci 2003; 116:813822. 